Fecha de recepción: abril 2020 Fecha de aceptación: mayo 2020 Versión final: junio 2020

\section{Diseño colaborativo en la sostenibilidad de los edificios. Una mirada holística a la creciente arquitectura ecológica en tiempos de pandemia COVID-19}

América María Alonso Ramírez ${ }^{(1)}$

\begin{abstract}
Resumen: Las acciones a nivel mundial por encontrar la sostenibilidad ecológica en el desarrollo de las actividades humanas se han incrementado de forma exponencial. Los crecientes cambios han promovido acciones por un diseño colaborativo, formando un equipo multidisciplinario que garantiza la toma de las decisiones ambientalmente responsables y económicamente viables en la creación y mantenimiento de los ambientes construidos. Esta creciente arquitectura ecológica toma auge a partir de la globalización de metodologías, estándares y sistemas de certificación incrementando la plusvalía de los diseños a nivel mundial. El nuevo usuario ve una armonía en diseño, materiales y espacio, ahora espera generar un impacto positivo al ambiente y garantizar su bienestar y salud.
\end{abstract}

Palabras clave: Sostenibilidad - diseño colaborativo - arquitectura ecológica - green building - sustainable design.

[Resúmenes en inglés y portugués en las páginas 192-193]

(1) Arquitecta y Magíster en diseño y construcción ecológicos (Universidad Rafael Landívar). Coordinadora del subprograma de diseño arquitectónico y urbano del Instituto de investigación y estudios superiores en arquitectura y diseño (INDIS), (Universidad Rafael Landívar).

La generación de espacios habitables es una necesidad humana que se remonta desde el origen del hombre como neandertal, donde se utilizaron cuevas con una función de resguardo y protección. Los aprendizajes pasados le han permitido evolucionar estos hábitats marcados por los aconteceres de la sociedad; civilizaciones antiguas como los mayas construyeron espacios no sólo habitables sino como una muestra de poder y expresión religiosa. Poco a poco se transformaron según los conocimientos y materiales de cada época llegando hasta la complejidad de los diseños arquitectónicos en el siglo veintiuno, que sorprenden con sus formas orgánicas, grandes escalas y diversidad de materiales y colores. Sin embargo la priorización por satisfacer las necesidades del hombre lo llevó a 
generar estas edificaciones sin importar las consecuencias negativas que dejaban, tanto en el entorno natural como en la salud y bienestar humanos.

En las últimas décadas la arquitectura y construcción ecológicas o frecuentemente conocidas como verdes, han tomado un importante auge en la sociedad influenciado por las aspiraciones de sostenibilidad ambiental propuestas desde finales del siglo pasado con el informe de Brundtland en 1987. Aunque lenta, su popularización en principio fue por los beneficios económicos que representaba. Pero gracias al incremento por la idea de contar con una mejor calidad de vida y establecer métodos ambientalmente sostenibles para la generación de las actividades cotidianas, su aceptación en la sociedad fluyó de forma exponencial. La creación de este tipo de edificaciones incrementó considerablemente en la última década a nivel global, sólo el WorldGBC (2019, p. 27) quien organiza los consejos de diseño y construcción verdes en el mundo, reportó en el 2019 la representación de esta organización en 69 países de los cuales 13 son latinoamericanos incluyendo Argentina y Guatemala. Además expuso que cuenta con más de 36,000 miembros activos garantizando así 2.9 billones de metros cuadrados construidos y operados de forma sostenible en todo el mundo. Esta nueva forma de diseñar y construir ha establecido metodologías que implementan estrategias de diseño colaborativo en equipos multidisciplinarios, para asegurar que cada aspecto fue propuesto en beneficio de las personas, el medio ambiente y la factibilidad económica.

La arquitectura ecológica genera grandes beneficios a los usuarios en aspectos que antes parecían indiscutibles como el obtener confort térmico en espacios interiores y los cuidados en la salud de los ocupantes, previendo la generación de enfermedades y promoviendo la productividad en las áreas de trabajo. Sin embargo el actual impulso de esta en los medios de comunicación la ha expuesto de forma superficial, como una tipología de diseño que implementa espacios vegetados como muros o cubiertas jardín. Inclusive la introducción de paneles solares en un proyecto hace creer a los usuarios que esto por sí sólo ya representa a una arquitectura sostenible. La desinformación popular no le hace justicia al trabajo y análisis que estos proyectos representan. Una mirada holística a esta creciente arquitectura puede informar cómo apoya a la sostenibilidad ecológica al mismo tiempo de mejorar la calidad de vida humana. Ahora más que nunca esto se percibe como indispensable ya que por temas de COVID-19, las construcciones actuales y futuras deberán garantizar la salud y bienestar de los usuarios además de funcionar como espacios habitables.

La presencia humana condiciona características en su entorno relacionadas a las comodidades adquiridas por el desarrollo de las actividades diarias; los espacios urbanos y habitados en general pueden crear condiciones climáticas y contextuales distintas a las naturales llegando a generar cambios en la interacción natural de los ecosistemas. La contaminación lumínica y de desechos, la disminución de áreas permeables que son responsables del ciclo hídrico natural, así como el acaparamiento desmedido de los recursos son sólo algunas de las problemáticas ambientales que hoy se viven en el mundo en consecuencia de las construcciones hechas por el hombre. En este sentido se debe entender el problema no sólo por el proceso de edificación sino también durante toda la vida útil del inmueble con su operación y mantenimiento, este último será tal vez el más importante por la prolongación de su uso. Por tanto, la arquitectura ecológica involucra nuevas construcciones así como las modificaciones en edificios existentes, donde sus actuales ocupantes tienen 
aspiraciones por una sostenibilidad ambiental. Una grandeza del ser humano es aprender de sus errores, y en este caso intentar corregir los aspectos negativos con los que estos inmuebles fueron concebidos. Esta arquitectura alcanza dicha sostenibilidad abordando dos factores principales: disminuyendo la contaminación ejercida tanto por la construcción como la operación de los edificios, y la implementación de mejores prácticas en la toma de decisiones relacionadas al manejo de los recursos.

La contaminación ambiental por ejemplo tiene muchas variantes y clasificaciones pero la más visible a nivel mundial tal vez sea la provocada por la generación de residuos sólidos. Según el World Bank (2018, p. 5, 29, 34) a nivel global el 45\% de todos los residuos terminan en un vertedero municipal y un angustiante $33 \%$ en espacios abiertos no controlados, lo que en el 2016 generó el 5\% del total de emisiones de gases efecto invernadero que contribuyen al ya tan conocido cambio climático. Esto considerando que no todos los desechos son recolectados para definir estas estadísticas. Sólo en Latinoamérica la cobertura de recolección es del $84 \%$ tomando en cuenta que en áreas rurales la mayoría no llega ni al 30\%. Las consecuencias negativas de esta problemática también abordan otros aspectos como la contaminación de los recursos hídricos del planeta. De forma trágica se observa como los ríos, lagos y mares se han llenado de grandes toneladas de basura en todo el mundo.

Para edificios de cualquier tipo de uso, la contaminación por desechos se genera tanto del proceso de edificación con los desperdicios de construcción así como la proveniente de la operación de los mismos cuando éstos se encuentran en uso. En ambos casos la sostenibilidad propuesta en la arquitectura ecológica presenta la planificación consiente de estrategias para establecer programas de reciclaje y concientización a los usuarios. Esta puede al menos ser regulada para evitar que todo termine en vertederos y aprovecharlo como recurso para utilizarse en procesos de reciclaje. Los desperdicios de construcción son un poco más complicados para tratar pero no imposibles de gestionar, y en la operación de los edificios existentes bastará con un breve análisis de las posibilidades para reciclaje de cada categoría de los desechos generados según las capacidades locales; a nivel mundial lo más común para reciclar es el papel y cartón, plásticos, vidrio y metales. Otra estrategia implementada en edificios existentes es la incorporación de políticas internas que fomenten la compra de materiales provenientes de procesos de reciclaje, reutilizados o que fueron creados con acciones favorables a la sostenibilidad ambiental en todos su proceso desde la manufactura hasta el transporte para la entrega final del producto. Esto apoya a cerrar un ciclo de vida idóneo del consumo y desechos de los materiales, evitando la extracción de la materia prima utilizada en sus fuentes naturales como lo es el caso del papel reciclado.

Otro aspecto para considerar es la contaminación lumínica generada por un diseño pobre que da una mala dirección o mal uso de la luz en resultado de la inapropiada aplicación de ésta, principalmente en espacios exteriores. La iluminación exterior provee al ser humano de seguridad y confort además de incrementar la productividad en un día. Sin embargo, el traspaso de esta en sitios adyacentes y el resplandor generado por los edificios en la noche, crea una abundante cantidad de problemas ambientales. Entre estos se pueden mencionar al efecto negativo en las actividades nocturnas como la caza realizada por especies animales, flora y fauna incapaces de ajustarse a variaciones temporales por no preservar la 
integridad de la noche, hasta la desorientación de aves migratorias que basan su curso en el cielo estrellado para guiarse (USGBC, 2013, p. 97-98). Para evitar estos cambios en el ciclo normal de la vida en los entornos urbanos es importante la medición de iluminación en los espacios exteriores con equipos especializados, proporcionando así el impacto lumínico de cada proyecto por individual. Regularmente estas mediciones son realizadas a un mínimo de ocho metros de distancia de la edificación. Otra estrategia ha sido el análisis de campo evaluando cada luminaria para determinar si la dirección y espectro de luz que emite es la más adecuada.

La contaminación del aire derivada de las emisiones de gases efecto invernaderos generados por los medios de transporte que utilizan los usuarios de un edificio, es otro factor importante para considerar en temas ambientales. En el caso de las nuevas construcciones, juega un papel principal las decisiones del equipo de planificación para un proyecto. Aquí deberá ser prioridad el fácil acceso al transporte público o su cercanía a circuitos de ciclovías para disminuir la generación de la contaminación por el transporte de los usuarios. No sólo la selección de ubicación para el edificio es transcendental, sino también el diseño de los diferentes accesos a este dónde la prioridad debería ser el peatón y disminuir la cantidad de usuarios que accedan en vehículo propio solos. En el caso de edificios existentes poco se puede hacer con temas de la ubicación pues, para bien o mal, esto ya fue definido en su concepción inicial. Sin embargo existen acciones que se han generado en torno a esta arquitectura sostenible, iniciando por conocer el medio de transporte y distancia recorrida por una buena muestra de ocupantes en el inmueble. Seguido de propuestas de parqueos preferenciales para aquellos que decidan compartir vehículo con otros compañeros laborales, en el caso de oficinas o campus universitarios por ejemplo.

Grandes problemáticas, que no involucran temas de contaminación, son consecuencia de las actividades humanas derivadas de la construcción de edificios o la operación de los mismos. En el caso específico del manejo del sitio, una de las más grandes preocupaciones es la destrucción de los ecosistemas conforme los espacios urbanos incrementan su tamaño, así como la erosión del suelo y la introducción de especies no nativas a estas áreas que terminan por complicar la vida que ya se estaba desarrollando en el lugar. Una arquitectura verdaderamente ecológica buscará mantener los ciclos naturales existentes del lugar, inclusive generando impactos positivos en los ecosistemas. La selección de plantas nativas para un diseño paisajista por ejemplo, no sólo mantiene la vida de las especies de animales e insectos que dependían sino también apoya al ahorro del agua de riego pues es vegetación que se encuentra adaptada al clima local. Estrategias más avanzadas involucran la generación de sistemas y prácticas con desarrollo de bajo impacto o LID por sus siglas en inglés. Estas utilizan el diseño y planificación en simultáneo con tecnologías para preservar y proteger los recursos naturales del ecosistema. Entre estos están los jardines de lluvia, cubiertas vegetadas, pavimentos permeables y las unidades de bioretención (U.S. HUD, 2003, p. 1, 14, 7-18, 29-30).

La adopción me mejores prácticas y políticas para la utilización de los recursos es un factor importante que afecta directamente al entorno ecológico. La reducción de los recursos hídricos para el abastecimiento en los espacios habitados, así como la generación de energía para la operación de los mismos. En el caso del agua según la UNESCO (2020, p. 20, 22-24) la demanda del recurso hídrico a nivel mundial ha incrementado su volumen 
seis veces en los últimos 100 años. Este incremento exponencial pone en riesgo el abastecimiento del mismo, estimando que para el año 2050 más de 685 millones de personas enfrentarán una crisis debido a la disminución de este recursos. En temas de sostenibilidad en edificios, la eficiencia del recurso es el mayor aporte que tiene esta industria a través de la implementación de artefactos sanitarios de bajo consumo hídrico. Por otra parte, la generación de energía ha sido un tema de preocupación global desde inicios del siglo veintiuno por la creciente demanda derivada del aumento poblacional mundial. Según proyecciones de la EIA (2019, p. 24, 27-29, 32, 150-151) se espera que para el año 2050 el consumo energético global incremente en más del 50\% del actual. Considerando que la principal fuente de energía son combustibles fósiles y orgánicos como la leña, no es sorpresa que se emitan grandes emisiones de $\mathrm{CO} 2$ contaminando el medio ambiente. Sin embargo en los últimos años la generación de energía ha disminuido la contaminación ambiental global gracias a los esfuerzos por países donde se han fomentado la eficiencia en el uso del recurso y la adopción de fuentes renovables para la generación del recurso. Estándares y certificaciones internacionales se han generado con la idea de delimitar los consumos máximos que los equipos eléctricos como luminarias, computadoras y artefactos de aire acondicionado deberían utilizar.

Los efectos negativos provocados en el medio ambiente por la generación de los edificios y espacios habitables para el ser humano son bastante claros. Si bien la arquitectura ecológica o sostenible busca mantener el equilibrio con la vida en el planeta, esta a su vez centra algunas acciones por garantizar el bienestar y salud humanas que fueron deterioradas en las últimas décadas. La constante innovación en el diseño arquitectónico desde la concepción de los proyectos hasta la selección de materiales, ha dejado por un lado conceptos básico que garantizaban el bienestar de los usuarios. Términos como la arquitectura vernácula suenan ahora tan ajenos a la arquitectura contemporánea en los espacios urbanos, pero en realidad se trata de edificaciones con materiales locales que buscaban el confort humano como prioridad. Si bien en diseño e innovación éstos no aportarán mucho, son funcionales y promueven el confort y salud para sus ocupantes.

Desde el mes de marzo en el año 2020 debido a la crisis mundial del COVID-19, la humanidad se vio en la necesidad de recurrir a la puesta en cuarentena de la mayor parte de la población por la facilidad de contagio del virus. Las restricciones de movilidad y el confinamiento en casa fueron algunas de las medidas que se tomaron desde un inicio, esto junto al cierre de fronteras y limitaciones de movilidad para el desarrollo de actividades cotidianas. Luego de permanecer por días, semanas y algunos hasta meses sin salir de sus hogares, es ahora donde los mejores diseños arquitectónicos salen a la luz pues los usuarios pueden expresar su completa satisfacción o insatisfacción por los espacios en los que habitan. El confort térmico aquí juega un papel principal en cuanto a percepciones. Si analizamos por ejemplo el caso de la ciudad ecuatoriana de Guayaquil, una de las más afectadas en Latinoamérica por la pandemia, la cual registró temperaturas superiores a los $32^{\circ} \mathrm{C}$ durante los primeros días de mayo siendo así los picos más altos de sus datos históricos (García, 2020). Aunque el drama mediático se ha focalizado en la cantidad de muertos de la ciudad y las complicaciones que esto genera, algunos expertos han expresado sus preocupaciones por el incremento de contagio por coronavirus en la población. En la ciudad de Guayaquil viven aproximadamente 2.3 millones de habitantes y las auto- 
ridades ecuatorianas han hecho mención en repetidas ocasiones que el incremento en las olas de contagios son porque en Ecuador "no hemos seguido con rigor estricto todas las medidas que se deben tomar para afrontar una emergencia de esta magnitud, ni las personas han hecho caso de las observaciones del gobierno [...]" (Millán, 2020). Sin embargo las condiciones de vivienda no son iguales para todos, según las estadísticas el 16.6\% de la población de Guayaquil vive en condiciones de hacinamiento en donde familias de hasta nueve integrantes comparten un espacio no mayor a los 60 metros cuadrados sin patio ni ventilación (Pesantes, 2020). Además las construcciones regularmente cuentan con una cubierta de lámina, un excelente transmisor de calor por conducción y en este caso las altas temperaturas lo hacen un medio para transmitir esa energía calorífica por convección al aire del espacio interior. Sumados estos tres factores: las altas temperaturas, el hacinamiento de personas en pequeños espacios sin ventilación adecuada y materiales constructivos que no aportan al confort térmico interior, es comprensible qué muchos de estos habitantes prefieren salir de sus hogares durante el día incrementando así sus posibilidades de contagio.

Sin embargo, este no es un evento aislado, ni ocurre tan sólo en viviendas de condiciones precarias. Una gran variedad de proyectos habitables que no consideraron las condiciones climáticas para el diseño y selección de materiales, ahora generan un gran descontento e insatisfacción a los usuarios quienes se ven obligados a permanecer en estos espacios que poco hacen por mejorar su bienestar. Y no sólo ocurre con la vivienda, también espacios de trabajo en condiciones inadecuadas provocan una disminución de productividad en sus ocupantes. Un correcto diseño arquitectónico deberá considerar las condiciones climáticas para la disposición del proyecto, así como la selección de formas y materiales que serán parte del envolvente. Actualmente existen una variedad de recubrimientos con propiedades térmicas específicas para el diseño exterior, hasta sistemas adecuados de ventilación e iluminación natural y artificial para los espacios interiores. Un cambio que ha tomado un auge en los últimos años ha sido la implementación de ventanas con propiedades de aislamiento térmico, que garantizan la iluminación interior sin la transferencia de calor. Ahora bien, esta problemática no sólo ocurre por temas de alza en temperaturas interiores, también existen materiales que por sus propiedades térmicas son adecuados para prevenir que el calor escape de los espacios y mantenerlos en temperaturas confortables aun cuando en el exterior se perciben más bajas. Analizando el mismo ejemplo de las ventanas con aislamiento, si en el espacio interior se cuenta con un sistema de calefacción artificial estas evitarán que la energía calorífica se escape fácilmente al exterior. Otro material que mantiene un buen equilibrio térmico y es muy popular en regiones del planeta con temperaturas más bajas es la madera.

Diversas decisiones son imprescindibles para generar un diseño de arquitectura sostenible. En términos de confort térmico no sólo la correcta selección del envolvente es parte de las medidas obligatorias de esta tipología, sino también factores externos provocados por la edificación que puedan apoyar a estos sucesos, tal es el caso del conocido efecto isla de calor. Este es un alza en la temperatura ambiental ocasionado por factores como las superficies no reflectivas en estacionamientos, caminos y cubiertas que absorben la radiación emitida por el sol durante el día. En consecuencia las áreas urbanas suelen manejar temperaturas más altas que los espacios rurales, variando entre $10^{\circ}$ y $15^{\circ} \mathrm{C}$ adicionales en 
el día, y durante la noche entre $5^{\circ}$ y $10^{\circ} \mathrm{C}$. Además del confort térmico, el efecto isla de calor afecta el bienestar y equilibrio ambiental así como aumenta el consumo energético por la necesidad de contar con equipos de aire acondicionado para refrescar los espacios interiores. Esto a su vez contribuye a la contaminación ambiental del planeta. El reincorporar espacios vegetados y permeables no sólo trae beneficios ambientales para los ecosistemas como ya fue mencionado antes, también permite contrarrestar los efectos negativos de esta problemática. Por otra parte, existen materiales altamente reflectivos y emisivos que pueden ayudar a evitar el efecto, como recubrimientos para cubiertas que con sus propiedades térmicas no sólo reflectan esta radiación sino también previene la transmisión de la energía al interior. Estos varían desde pinturas para cubiertas y fachadas hasta membranas prefabricadas (EPA, 2008, p. 1-2, 6-9, 12, Ch1 p. 13-15, Ch4 p. 1, 5-6).

Dejando por un lado el tema superficial de las construcciones, el bienestar y salud humanos no sólo se mide por las percepciones de los usuarios sino también por las acciones que, imperceptibles a estos, están promoviendo el cuidado de la vida que habita dentro de estos espacios edificados. Esto es parte de las acciones de la arquitectura ecológica o sostenible descubrir que el diseño, la construcción y operación de edificios van más allá de lo observado por los ojos, e involucra factores internos y externos que darán al ocupante una experiencia completa de bienestar. Principalmente trae grandes beneficios en los tiempos de ocupación y uso de las edificaciones.

La salud de los seres humanos se ha visto comprometida en las últimas décadas por la constante contaminación del aire generada en el desarrollo de las actividades humanas diarias. Estudios científicos han demostrado que dicha exposición a estos agentes pueden provocar daños considerables como enfermedades cardiovasculares y respiratorias hasta efectos en el sistema nervios como deficiencias para aprender y pérdida de memoria (EPA, 2010, p. 3, 5, 14, 18, 20, 26). Según la Organización Mundial de la Salud (2006, p. 16, 33 , 48-49) al año mueren 1.5 millones de personas en todo el mundo, como consecuencia de problemas respiratorios relacionados directamente a este tipo de contaminación. Además señala que la exposición a estas partículas contaminantes es la responsable del 12\% de las enfermedades pulmonares crónicas y un $20 \%$ obtuvieron asma por permanecer en interiores húmedos, con ácaros de polvo y hongos que ocasionaron alergias. Así mismo, vincula enfermedades cardiovasculares asociadas a la contaminación del aire por una exposición prolongada al plomo, el cual puede incrementar la presión arterial.

En las áreas urbanas el 90\% de las personas pasan la mayor parte de su tiempo en espacios interiores ya sea por vivienda, trabajo o recreación, en donde la exposición a estos agentes contaminantes puede ser hasta 50 veces más alta que la generada en el exterior. Cientos de partículas químicas y biológicas se encuentran circulando en el aire de los ambientes construidos, sin embargo la falta de información y conocimiento al respecto ha provocado que estas problemáticas sean desconocidas por los usuarios. Tal vez lo más conocido al respecto es el síntoma de edificio enfermo o SBS por sus siglas en inglés, el cual describe un espectro de quejas y malestares físicos específicos por los ocupantes de un edificio determinado en una proporción mayor a la que sería razonable (EPA, 2001, p. 2, 4-8). Para prevenir la contaminación del aire es importante establecer protocolos que disminuyan la introducción de estos agentes en los ambientes interiores. La arquitectura ecológica que verdaderamente busca preservar el bienestar humano ha desarrollado estrategias como la 
incorporación de rejillas y sistemas ranurados en los ingresos peatonales para prevenir la entrada de contaminación exterior a través de los usuarios en su acceso al inmueble.

Determinar la fuente de contaminación en el interior también es importante pues algunos productos como las placas de yeso, las pinturas, paneles de melamina, tapicerías, adhesivos e inclusive cortinas emiten compuestos orgánicos volátiles o VOC por sus siglas en inglés, los cuales deterioran la calidad del aire en términos sensoriales como olores e irritación de ojos y vías respiratorias, así como la pérdida de productividad ocasionada por una exposición constante (Wolkoff, 1995, p. 11, 13, 17, 19). En este sentido, promover el uso de materiales y mobiliario que estén libres de cualquier agente contaminante que pueda poner en riesgo la salud de los ocupantes, y de preferencia se encuentre avalado por alguna otra entidad, será una de las mejores prácticas para crear espacios saludables. En el caso de las pinturas para interiores por ejemplo, el mercado actual presenta una variedad de opciones que no emiten VOCs. Adicional a esto, una adecuada ventilación natural o artificial de los espacios interiores ayudará a preservar la salud de los usuarios, garantizando así que las renovaciones de aire adecuadas se mantengan. Una gran variación de entidades y estándares internacionales han propuesto desempeños ideales y distribuciones adecuadas en los espacios para preservar esta calidad del aire. En el caso de los sistemas de acondicionamiento artificial pueden determinar con datos específicos las renovaciones de aire generadas, tasas mínimas para el caudal de ventilación exterior, así como los procedimientos para el adecuado mantenimiento de los equipos. En el caso de la ventilación natural siendo más complejas las mediciones, existen normativas que presentan promedios establecidos para la distribución en los espacios interiores. Según ANSI y ASHRAE (2016, p. 2, 5, 11-13, 19, 21-22) por ejemplo expone que la distancia máxima en un espacio con ventanas en un solo lado, en relación con la pared opuesta no debe superar dos veces la altura de piso a techo. Siempre es recomendado diseñar pensando en los flujos de ingreso y salida del aire en los espacios, además de no contar con agentes contaminantes en las cercanías para evitar el ingreso a los ambientes. Ahora por la creciente preocupación del COVID-19, las renovaciones de aire toman una mayor importancia para garantizar la salud de los ocupantes en cualquier ambiente interior. Muchos estudios se han realizado exponiendo cómo éstas apoyan a la regulación de la humedad en los espacios interiores. Esta última en muchas ocasiones es la responsable de que virus, bacterias y hongos permanezcan y habiten en las edificaciones, pues con mucha o muy poca humedad muchos de estos microorganismos y partículas logran sobrevivir. Entidades con ASHRAE están realizando pruebas para determinar los parámetros adecuados que garanticen la calidad del aire sin transmitir el coronavirus a otros espacios ni poner en riesgo a los ocupantes. La arquitectura ecológica, verde o sostenible busca al final mantener el equilibrio ambiental, humano y económico en cada proyecto sin importar su estado, desde nuevas construcciones hasta edificios ya existentes. Los crecientes proyectos generados en torno a estas metodologías han aportado grandes acciones para el medio ambiente y al uso de nuevas tecnologías, muchas reconocidas o avaladas por entidades internacionales a través de certificaciones que validan dichos procesos. La popularización de estas ha incrementado en las últimas décadas generando así una gran variedad de opciones que, si bien las acciones mencionadas con anterioridad no se encuentran condicionadas a un proceso de certificación específico, al obtener un sello o certificación internacional que valide los 
procesos le traerá reconocimientos a nivel local e internacional. No hay un gran mérito en decir que acciones de sostenibilidad se están realizando si quien lo genera es quien lo evalúa y lo presenta. Entre las más reconocidas a nivel mundial se pueden mencionar a LEED, EDGE o WELL, esta última evalúa más acciones por preservar el bienestar humano en espacios interiores. También se puede destacar iniciativas como el Living Building Challenge, BREEAM o Passivhaus, inclusive locales como es el caso de la certificación CASA en Guatemala, otorgada por el Guatemala GBC.

La sostenibilidad en el ámbito del diseño y construcción será posible con la implementación de un grupo multidisciplinario, que en acciones para la generación de un diseño colaborativo busque preservar la ecología y salud de los humanos al mismo tiempo de garantizar la factibilidad económica. Esta nueva metodología ya no es un trabajo aislado de arquitectos, diseñadores e ingenieros ahora se busca integrar agrónomos, biólogos, economistas y otras profesiones que puedan evaluar cada aspecto desde su ámbito de especialización en los proyectos y generar en conjunto propuestas innovadoras. Ya no sólo por sus apariencias y las experiencias de los usuarios, sino por las tecnologías y nuevos conocimientos que se integran permitiendo que la vida en el planeta se siga generando. Para promover dichas acciones es importante la participación de los ocupantes que ahora gracias a la globalización, el internet y los medios de comunicación cada vez es más fácil reconocer estas buenas prácticas a nivel mundial y local. Demandar mejores espacios, mejores diseños y arquitectura ecológica en las áreas urbanas permitirá que cada día se perciba con mayor normalidad estas iniciativas, impulsando a quienes aún no lo generan a que cambien las antiguas metodologías y en conjunto se busque preservar la vida en todos los sentidos. Entender que hacer ecología es más que colocar muros o cubiertas verdes, es comprender el desarrollo de todos y todo lo que habita en el planeta cuando de diseño y construcción arquitectónica se habla. Pero sobre todo, que el conocimiento es control en las decisiones que se toman, y que como usuarios de estos espacios habitables informarse sobre el funcionamiento de las edificaciones permitirá prevalecer nuestro bienestar.

\section{Listado de referencias bibliográficas}

ANSI \& ASHRAE. (2016). Ventilation for Acceptable Indoor Air Quality. Atlatnta: ASHRAE. García, A. (11 de mayo 2020). Guayaquil registra temperaturas por encima de lo normal para mayo, en la transición de la etapa lluviosa a seca. El Comercio. Recuperado de: https:// www.elcomercio.com/actualidad/guayaquil-temperaturas-transicion-etapa-lluviosa.html

Millán, A. (2 de abril de 2020). Coronavirus: ¿por qué Ecuador tiene el mayor número de contagios y muertos per cápita de covid-19 en Sudamérica?. BBC NEWS MUNDO. Recuperado de: https://www.bbc.com/mundo/noticias-america-latina-52036460

Pesantes, K. (4 de abril de 2020). Hacinamiento y pobreza dificultan la cuarentena en Guayaquil. PRIMICIAS. Recuperado de: https://www.primicias.ec/noticias/sociedad/ guayaquil-hacinamiento-pobreza-cuarentena/

UNESCO, (2020). The United Nations World Water Development Report 2020. Water and climate change. Paris: United Nations Educational, Scientific and Cultural Organization. 
U.S. Department of Housing and Urban Development. (2003). The Practice of Low Impact Development. Maryland: HUD.

U.S. Environmental Protection Agency. (2001). Healthy Buildings, Healthy people: a vision for the 21st. Century. Washington DC: EPA.

U.S. Environmental Protection Agency. (2008). Reducing urban heat islands: Compendium of strategies. Washington DC: EPA.

U.S. Environmental Protection Agency. (2010). Our Nation's Air, Status and trends through 2008. Carolina del Norte: EPA.

U.S. Energy Information Administration. (2019). International Energy Outlook 2019 with projections to 2050. Washington DC: EIA.

USGBC, (2013). LEED Reference Guide for Building Operations and Maintenance. Washington DC: U.S. Green Building Council.

Wolkoff, P. (1995). Volatile Organic Compounds Sources, Measurements, Emissions, and the Impact on Indoor Air Quality. En Official Journal of the International Society of Indoor Air Quality and Climate: Copenhagen, Dinamarca.

World Green Building Council. (2019). Building a better future, Annual report 2018/19. Toronto: The World Green Building Council.

\begin{abstract}
Actions worldwide to find ecological sustainability in the development of human activities have increased exponentially. The growing changes have promoted actions for collaborative design, forming a multidisciplinary team that guarantees environmentally responsible and economically viable decision-making in the creation and maintenance of the built environments. This growing ecological architecture is booming from the globalization of methodologies, standards and certification systems, increasing the added value of designs worldwide. The new user sees a harmony in design, materials and space, now he hopes to generate a positive impact on the environment and guarantee his well-being and health.
\end{abstract}

Keywords: Sustainability - collaborative design - ecological architecture - green building - sustainable design.

Resumo: As ações em todo o mundo para encontrar sustentabilidade ecológica no desenvolvimento de atividades humanas aumentaram exponencialmente. As mudanças crescentes promoveram ações para um design colaborativo, formando uma equipe multidisciplinar que garante a tomada de decisões ambientalmente responsáveis e economicamente viáveis na criação e manutenção dos ambientes construídos. Essa crescente arquitetura ecológica está crescendo desde a globalização de metodologias, padrões e sistemas de certificação, aumentando o valor agregado dos projetos em todo o mundo. $\mathrm{O}$ novo usuário vê uma harmonia em design, materiais e espaço, agora espera gerar um impacto positivo no meio ambiente e garantir seu bem-estar e saúde. 
Palavras chave: Sustentabilidade - design colaborativo - arquitetura ecológica - construção verde - design sustentável.

[Las traducciones de los abstracts fueron supervisadas por el autor de cada artículo] 